\title{
Intrinsic Properties Analysis of Multiproteases System from Marine Bacteria by Inhibitor-Subsatrate Immersion Zymography
}

\author{
Dan Liu ${ }^{1}$, Cui-Ling Wu ${ }^{1}$, Xing-Hao Yang ${ }^{1}$, Ri-Bang Wu ${ }^{1}$, Jiang Zhang ${ }^{1}$, Jia-Heng Huang ${ }^{1}$, \\ Bin-Qiang Liao", Fei Bian', Hai-Lun He ${ }^{1, *}$ \\ ${ }^{1}$ School of Life Sciences, State Key Laboratory of Medical Genetics, Central South University, Changsha, China \\ ${ }^{2}$ Biotechnology Research Center, Shandong Academy of Agricultural Sciences, Jinan, China
}

Email address:

helenhe@csu.edu.cn (Hai-Lun He)

${ }^{*}$ Corresponding author

\section{To cite this article:}

Dan Liu, Cui-Ling Wu, Xing-Hao Yang, Ri-Bang Wu, Jiang Zhang, Jia-Heng Huang, Bin-Qiang Liao, Fei Bian, Hai-Lun He. Intrinsic Properties Analysis of Multiproteases System from Marine Bacteria by Inhibitor-Subsatrate Immersing Zymography. American Journal of BioScience. Vol. 4, No. 3, 2016, pp. 20-27. doi: 10.11648/j.ajbio.20160403.11

Received: May 11, 2016; Accepted: June 20, 2016; Published: June 21, 2016

\begin{abstract}
Based on digital image analysis techniques and inhibitor-substrate immersing zymography, intrinsic properties of each active component in the enzymatic system secreted by marine bacteria were studied. This method provides an easy way to characterize the proteases in situ, which can be further verified by Mass spectrometry. Compared to the Folin phenol method, a traditional method used to determine proteases activities, the inhibitor-substrate immersing zymography method coupled with digital image analysis used in this study could determine caseinolytic activity and measure gelatinolytic activity at the same time. The effect on activities of extracellular proteases by inhibitor (phenylmethylsulfonyl fluoride or 1, 10-Phenanthroline) can be quantified by gray value changes of the corresponding band after electrophoretic separation. Because of its high throughput, great sensitivity, and convenient operation, inhibitor-substrate immersing zymography can be used to demonstrate the natural diversity of protein hydrolases and multienzyme expression systems. Thus, it is an effective approach to study the functional proteomics of proteases secreted by marine bacteria.
\end{abstract}

Keywords: Gital Image Analysis, Inhibitor-Substrate Immersing Zymography, Extracellular Protease, Multienzyme, Marine Bacteria

\section{Introduction}

Proteases are also known as peptidases that hydrolyze the peptide bonds of proteins [1]. Proteases are widely distributed in all biological organisms and perform different physiological functions. Proteases have been one of the most widely-used enzymes in the world. They occupy a pivotal position with respect to their applications in both physiological and commercial fields. Proteases account for about $60 \%$ of the total worldwide sale of enzymes [2]. According to the catalytic mechanism, the proteases are divided into aspartic proteases, glutamic proteases, cysteine proteases, metalloproteases, serine proteases, threonine proteases and unknown catalytic types of protease in the
MEROPS database [3]. The vast diversity of proteases, with respect to the specificity of their actions, has attracted worldwide attention to exploit novel proteases and its new applications [4]. In particular, microbes serve as an excellent source of enzymes because of their rapid growth, the simple requirement for cultivation and the ease genetic manipulation to generate new enzymes. To meet current world demands, more and more microbial proteases have been discovered. Most of these proteases were identified from terrestrial microorganisms, but less from marine [5]. Due to the biodiversity and specificity of the marine environment, marine microorganisms may become important sources for the 
selection of novel proteases.

The marine microorganisms, which have ecological significance in nitrogen cycling, are responsible for degradation of organic biopolymers and redistribution of organic matter among different components of the ocean ecosystem [6]. Since proteins are the most important component of marine organic polymers, protease-producing bacteria can play vital roles in decomposing of organic polymers [7]. Moreover, hydrolysis of proteins requires the collaboration of relevant enzymes [8]. Therefore, the diversity of enzymes in their natural habitats needs to be elucidated for rational design of protease cocktails with higher degrading efficiency. Methods have been developed to detect active proteases present in whole extracts, such as protease zymography $[9,10]$. Protease inhibitors are molecules that inhibit the function of proteases by combining with the protease active site or allosteric site, such as Cysteine protease inhibitors, Metalloprotease inhibitors, Serine protease inhibitors $[11,12]$. Protease inhibitors may be helping us determine the types of protease based on the mechanism of inhibition. Traditionally, protease inhibition assays generally couple a biochemical test for proteolysis with purified enzymes. However, the operation of purified enzymes is tedious and expensive. Therefore, it is highly desirable to find more effective method to accurately and rapidly detect the type of extracellular proteases in situ.

In this study, we explored the modification of the protease detection method described in our previous studies [13] and coupled it with an inhibitor experiment for detecting the type of protease. The characteristics of extracellular proteases from different marine bacteria were determined by this method. This technique will provide a useful and rapid method of detecting protease types, enzymatic characteristics and bacterial multi-protease system, and will have wide applications beyond proteolytic enzymology.

\section{Materials and Methods}

\subsection{Bacteria Strains}

The bacteria strains used in this study were isolated from the inshore environment of Bohai Sea or the South China Sea They were identified as the genera, Vibrio and Bacillus by $16 \mathrm{~S}$ rDNA sequences analysis and have been submitted to GenBank. The strains investigated in this study are listed in Table 1.

Table 1. Bacteria strains used in this study.

\begin{tabular}{llll}
\hline Strains & $\begin{array}{l}\text { GenBank } \\
\text { No. }\end{array}$ & Closest Species & $\begin{array}{l}\text { Similarity } \\
(\%)\end{array}$ \\
\hline $\begin{array}{l}\text { Pseudoalteromon } \\
\text { as } \text { sp. CSN423 }\end{array}$ & KP789967 & $\begin{array}{l}\text { Pseudoalteromonas } \text { sp. } \\
\text { BSi20669 }\end{array}$ & 99 \\
$\begin{array}{l}\text { Vibrio } \text { sp. } \\
\text { SQS2-3 }\end{array}$ & KF220487 & $\begin{array}{l}\text { Vibrio diabolicus } \\
\text { CW-9-11-1 }\end{array}$ & 99 \\
$\begin{array}{l}\text { Bacillus } \text { sp. } \\
\text { SQN6-1 }\end{array}$ & KF274051 & $\begin{array}{l}\text { Bacillus baekryungensis } \\
\text { XJSL3-2 }\end{array}$ & 98 \\
\hline
\end{tabular}

\subsection{Cultivation of Protease-Producing Bacteria}

The protease-producing strains were cultured at $15^{\circ} \mathrm{C}$ with shaking at $200 \mathrm{rpm}$ in $500 \mathrm{ml}$ flasks containing $50 \mathrm{ml}$ of fermentation broth ( $2 \%$ corn powder, $1 \%$ bean powder, $2 \%$ wheat bran, $0.1 \% \mathrm{CaCl}_{2}, 0.4 \% \mathrm{Na}_{2} \mathrm{HPO}_{4}$, and $0.03 \% \mathrm{KH}_{2} \mathrm{PO}_{4}$, prepared with sea water) [14]. The culture supernatant was collected by centrifugation $\left(10,000 \times \mathrm{g}, 4^{\circ} \mathrm{C}, 20 \mathrm{~min}\right)$ after 96 hours incubation and stored at $-20^{\circ} \mathrm{C}$ for electrophoresis analysis.

\subsection{Effect of Inhibitors on Protease Activity Assay}

The culture supernatant was diluted appropriately with 50 $\mathrm{mM}$ Tris- $\mathrm{HCl}(\mathrm{pH} 8.0)$, and was pre-incubated with was pre-incubated with $1.0 \mathrm{mM}$ phenylmethylsulfonyl fluoride (PMSF, Sigma, USA) or $1.0 \mathrm{mM} \mathrm{1,10-Phenanthroline} \mathrm{(OP,}$ Sigma, USA) at $37^{\circ} \mathrm{C}$ for $20 \mathrm{~min}$ [15]. After incubation, the residual proteolytic activity to casein was determined according to the method described by Zhou [15]. One unit of enzyme activity was defined as the amount of enzyme that catalyzed the formation of $1 \mu \mathrm{g}$ tyrosine per minute. The activity of samples without inhibitors was set $100 \%$ activity as control. All experiments were done in triplicate.

\subsection{In Situ Demonstration of Inhibitor-Substrate Immersing Zymography}

Electrophoresis was performed as our previous studies with a Bio-Rad Mini Protean 3 electrophoresis apparatus (Hercules, CA, USA). Ten microliter samples with proper dilution or concentration were mixed with the loading buffer (60 mM Tris- $\mathrm{HCl}$ (pH 6.8), 25\% glycerol, and $0.1 \%$ bromophenol blue) at the ratio of $4: 1$ and placed directly in the wells. Electrophoresis was carried out at $4{ }^{\circ} \mathrm{C}$ with constant voltage $(120 \mathrm{~V})$ for about $1.5 \mathrm{~h}$ until the bromophenol blue dye reached the bottom. After electrophoresis, the gels were washed three times with Triton $\mathrm{X}-100(2.5 \%)$ for $15 \mathrm{~min}$ at $10^{\circ} \mathrm{C}$ to remove SDS, and subsequently washed twice for $20 \mathrm{~min}$ with $50 \mathrm{mM}$ Tris $\mathrm{HCl}$ buffer [13]. The substrate solution of $0.2 \%$ gelatin or $0.2 \%$ casein (w/v, prepared by $50 \mathrm{mM}$ Tris-HCl buffer, $\mathrm{pH} 7.5$ ) was incubated at $37^{\circ} \mathrm{C}$ for $10 \mathrm{~min}$. Then the protease inhibitor (PMSF or OP) with the final concentration $1.0 \mathrm{mM}$ was added to the substrate solution respectively. The gel stripe was cut separately by each lane and following immersed in each of pre-warmed substrate solution with inhibitor and kept at $37^{\circ} \mathrm{C}$ for reaction $1 \mathrm{~h}$. After washing, the gels were stained with $0.1 \%(\mathrm{w} / \mathrm{v})$ Coomassie Brilliant Blue R-250 (Sangon, Shanghai Ltd, China.) for $3 \mathrm{~h}$ followed by destaining with $30 \%$ ethanol and $70 \%$ acetic acid solution until clear bands indicating proteolytic activity became visible.

\subsection{Protease Sequencing by Tandem Mass Spectrometry}

The sample treatment is following commonly used protocol [16]. Protease band of interest were excised from Coomassie-stained SDS-polyacrylamide gels and digested in 
situ with trypsin (Sigma, USA) in digestion buffer (ammonium bicarbonate $100 \mathrm{mM}, \mathrm{pH} 8.5$ ). The peptides from the digestion were extracted out with acetonitrile, and completely dried down in a SpeedVac device (Thermo Fisher, USA). Then the dried sample was redissolved in sample solution ( $2 \%$ acetonitrile $97.5 \%$ water, $0.5 \%$ formic acid). The protein solution was reduced by DTT (Sigma, USA) and all Cysteine residues alkylated by iodoacetamide and cleaned. And then a dissolved peptide sample was analyzed by a NanoLC-ESI-MS/MS system.

NanoLC-ESI-MS/MS analysis of digested protein sample is carried out by a high pressure liquid chromatography (HPLC) system (Agilent, USA) with a $75 \mu \mathrm{m}$ inner diameter $8 \mathrm{~cm}$ in length reverse phase $\mathrm{C} 18$ column. The particle size of the $\mathrm{C} 18$ was $3 \mu \mathrm{M}$ with pore size of $300 \ddot{A}$. The HPLC Solvent A was $97.5 \%$ water, $2 \%$ acetonitrile, $0.5 \%$ formic acid. HPLC Solvent B was $9.5 \%$ water, $90 \%$ acetonitrile, $0.5 \%$ formic acid The gradation time was 60 minutes from $2 \%$ to $90 \%$ solvent B The column flow rate was around 800 nanoliter per minute after splitting. Typical injection volume was $3 \mu \mathrm{l}$. The HPLC system is on-line coupled with an ion trap mass spectrometer (LCQ DECA XP PLUS, Thermo) in a way a sample eluted from HPLC column is directly ionized by an electrospray ionization (ESI) process and enter into the mass spectrometer. The ionization voltage is often optimized each time and normally in a range of $1.2 \mathrm{kV}-1.8 \mathrm{kV}$. The capillary temperature is set at $110^{\circ} \mathrm{C}$. The mass spectrometer is set at the data-dependent mode to acquire MS/MS data via a low energy collision induced dissociation (CID) process. The mass spectrometric data is used to search against the most recent non redundant protein database (NR database, $\mathrm{NCBI)} \mathrm{with}$ ProtTech's ProtQuest software suite.

\subsection{Quantitation Protease Activity Using Inhibitor-Substrate Immersing Zymography}

The gels were scanned using the transmitted light in an Epson Perfection V500 scanner (Seiko Epson, Japan). The bands' digital information was acquired mainly from the peaks generated by utilizing ImageJ software (National Institutes of Health, USA) and extracted quantitatively [17]. The peak area values, corresponding to the gray values of each band, were calculated by the method of peak volumes [13]. All experiments under the same condition were carried out in triplicate, and the mean values were analyzed with the Origin Pro 8.0 software (Origin Lab, USA). Setting the gray values of the original band to $100 \%$ intensity under the same experimental conditions, other relative intensity values can be calculated by it. The relative intensity of the band (the gray value) reflected the relative activity of extracellular proteases.

\section{Results and Discussion}

\subsection{Profiles of Trypsin and Collagenase by Inhibitor-Substrate Immersing Zymography}

To confirm whether the inhibitor-substrate immersing zymography was feasible or not, trypsin and collagenase were used for detected the residual activities. Trypsin is a typical serine protease, which was identified by their sensitivity to the phenylmethylsulfonyl fluoride (PMSF). Collagenase is a typical metalproteases that was inhibited with 1, 10-phenanthroline (OP). After non-reducing electrophoresis, the gel was incubated with substrate and inhibitor, OP and PMSF. The activity of trypsin was completely inhibited by PMSF, but not affected by OP (Fig. 1). The results showed that the method inhibitor-substrate immersing zymography was feasible to determine the profiles of proteases.

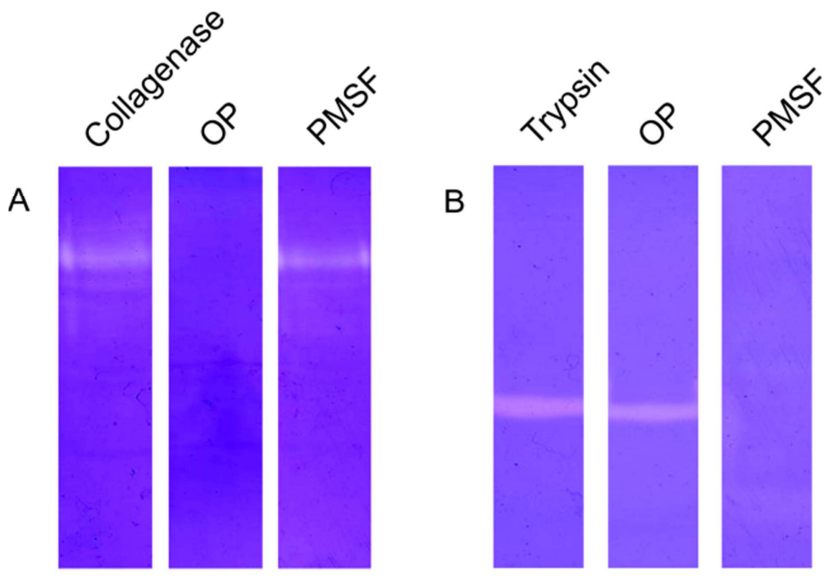

Fig. 1. The profiles of trypsin and collagenase $(1 \mathrm{mg} / \mathrm{ml})$ visualized by inhibitor-substrate immersing zymography (Inhibitor, $1 \mathrm{mM}$ PMSF or OP, Substrate, $0.1 \%$ gelatin). (A) The activity of collagenase in inhibitor-gelatin immersing zymography. (B) The activity of trypsinin inhibitor-gelatin immersion zymography.

\subsection{Demonstration and Quantitative Analysis of Extracellular Proteases from Marine Bacteria}

It has been reported that Pseudoalteromonas species are the predominant protease-producing groups in cultured marine bacteria [18] and show high protease-producing ability in the marine environment [15]. Moreover, Vibrio species are natural habitants in the aquatic environment and play important roles in maintaining the aquatic ecosystem. Also, the most common and widely used bacteria for industrial proteases belong to the genus Bacillus [19, 20]. For these reasons, we chose Pseudoalteromonas, Vibrio and Bacillus strains to clarify their extracellular protease profiles by inhibitor-substrate immersing zymography.

\subsubsection{Detection of Extracellular Protease Activities from Pseudolateromonas sp. CSN423}

Gelatin immersion zymography following SDS-PAGE resulted in detection of six major proteolytic bands of Pseudolateromonas sp. CSN423 (Fig. 2A). The proteases were distinguished by numbers according to the activities and the putative molecular variants, named E423-1, E423-2, E423-3, E423-4, E423-5, E423-6 and E423-7 from top to bottom, respectively. The zymographic detection of E423-1, E423-2 and E423-3 was sensitive to OP. This result indicated that these enzymes were metalloproteases. On the other hand, 
E423-4, E423-5, E423-6 and E423-7 were completely inhibited by PMSF, indicating these enzymes were serine protease (Fig. 2A). Only three obvious bands were separated when using casein as substrate. Because of these gel stripes were cut from the same gel slice, these three bands corresponded to E423-1, E423-2, and E423-3, which was further confirmed with similar inhibitor results. It is important to note that, interestingly, PMSF did not inhibit protease activity of E423-1, E423-2 and E423-3, but contributed to the enzyme activity.

As shown in Fig. 2B, the extracellular protease activity of Pseudolateromonas sp. CSN423 was strongly inhibited by OP from the results of Folin phenol method. The activity was reduced by $86.2 \%$ in the presence of $1 \mathrm{mM}$ OP. However, $1 \mathrm{mM}$ PMSF had no significant inhibitory effect on the activities of proteases secreted by Pseudolateromonas sp. CSN423. Meanwhile, the bands' digital information acquired mainly from the inhibitor-substrate immersion zymography were calculated by ImageJ $1.43 \mathrm{u}$ software (NIH, USA) and extracted quantitatively the intensity of protease bands were added together as the total extracellular enzyme activity. When casein was used as a substrate, similar results were obtained. The activities of crude enzymes from Pseudolateromonas sp. CSN423 were completely inhibited by OP, but PMSF has no effect on enzyme activity. However there were very different results when using gelatin as substrate. The bands' digital information showed that there were about $45 \%$ residual enzyme activities in the presence of $1 \mathrm{mM}$ OP and $55 \%$ residual enzyme activities in the presence of $1 \mathrm{mM}$ PMSF, respectively (Fig. 2B). The reason was that some proteases, such as E423-4, E423-5, E423-6 and E423-7, could not be detected on casein immersion zymography but can be detected on gelatin immersing zymography. And the activities of these four enzymes were undetectable by Folin phenol caseinolytic analysis.

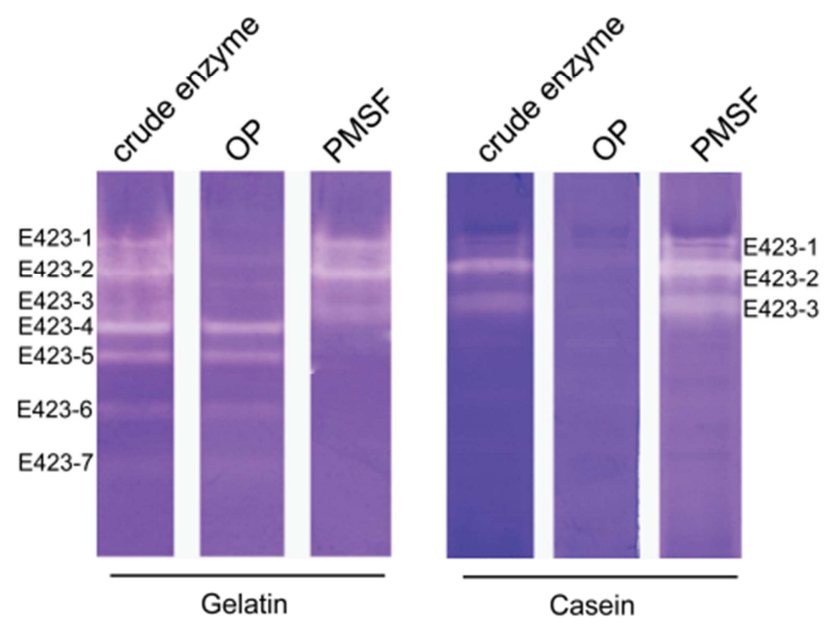

A

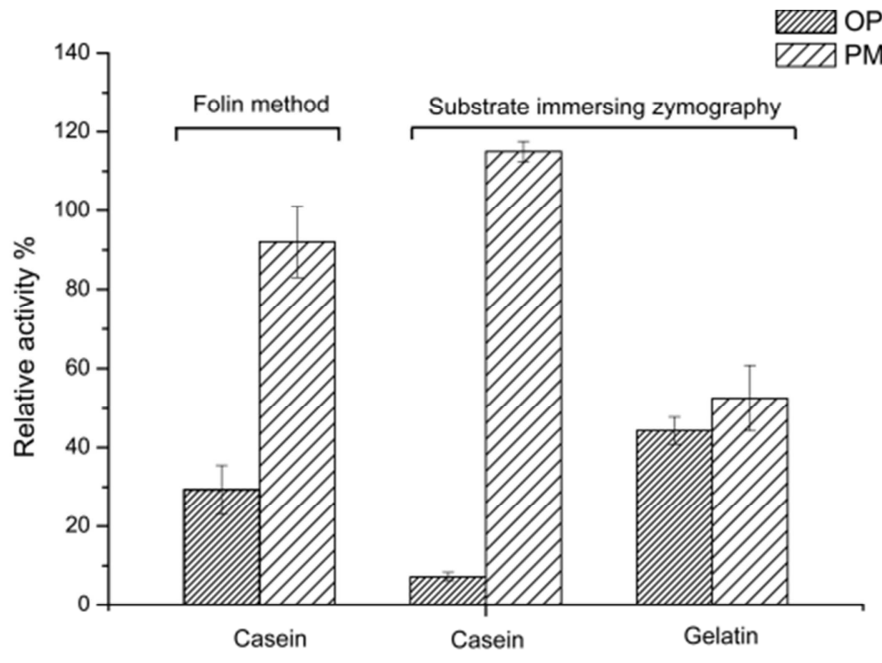

B

Fig. 2. The effect of inhibitors on proteases from the crude extract of Pseudolateromonas sp. CSN423. (A) Inhibitor-substrate immersing zymographic detection of Pseudolateromonas sp. CSN423 proteases in culture. The activities were tested with both casein and gelatin (0.1\%) as the substrate. The concentration of inhibitors during both sample treatment and gel slice incubation was $1 \mathrm{mM}$. (B) Compared analysis inhibition of proteases from the crude extract of Pseudolateromonas sp. CSN423 by OP or PMSF using Folin phenol method and inhibitor-substrate immersing zymography method. The relative activities were calculated by quantitative extraction of the gray values of the bands on zymogram.

\subsubsection{Detection of Extracellular Protease Activities from Bacillus sp. SQN6-1}

It was very clear that EN6-1, EN6-2, EN6-3, EN6-4 and EN6-6 were serine protease and EN6-5 was metalprotease secreted by Bacillus sp. SQN6-1 from the inhibitor-gelatin immersion zymography (Fig. 3A). But when casein was used as the substrate, only one broad proteolytic band of EN6-5 was observed. These results suggested that proteases EN6-1, EN6-2, EN6-3, EN6-4 and EN6-6 were more sensitive to gelatin than casein. EN6-5 was the widest band whether it was in the casein or gelatin substrates, so EN6-5 was the mainly Bacillus sp. SQN6-1 proteases in culture.

The activities of the proteases from Bacillus sp. SQN6-1 culture were inhibited by both $1 \mathrm{mM}$ PMSF and $1 \mathrm{mM}$ OP and were more sensitive to OP by Folin phenol caseinolytic analysis. Just like the results were determined based on digital image analysis techniques coupled with casein immersing zymography. The residual activity of Bacillus sp. SQN6-1 culture was $37 \%$ when $1 \mathrm{mM}$ PMSF was added. On the other hand, the activity of Bacillus sp. SQN6-1 culture was almost completely inhibited by $1 \mathrm{mM}$ OP (Fig. 3A). However when gelatin was used as substrate, several bands sensitive to PMSF appeared on the zymogram (Fig. 3A). Therefore, the bands' digital information showed about $18 \%$ residual activity with 1 mM OP treatment (Fig. 3B). 

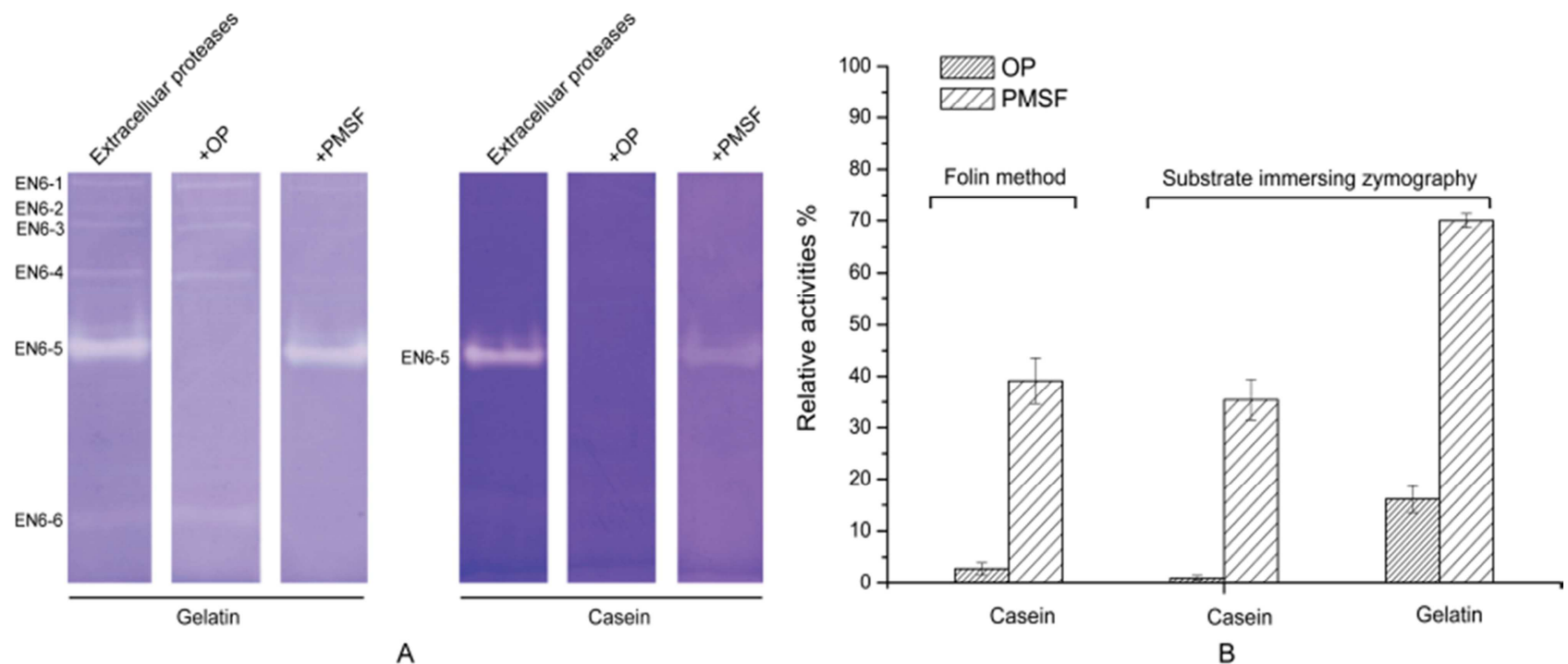

Fig. 3. The effect of inhibitors on proteases from the crude extract of Bacillus sp. SQN6-1. (A) Inhibitor-substrate immersing zymographic detection of Bacillus $s p$. SQN6-1 proteases in culture. After non-reducing SDS-PAGE, each lane was cut separately and incubated with various substrates (gelatin or casein) and inhibitors (OP or PMSF), and then the protease activities were determined. (B) Compared analysis inhibition of proteases from the crude extract of Bacillus sp. SQN6-1 by OP or PMSF using Folin phenol method and inhibitor-substrate immersion zymography method.

\subsubsection{Detection of Extracellular Protease Activities from Vibrio sp. SQS2-3}

Three different activity bands from Vibrio sp. SQS2-3, named EV-1, EV-2 and EV-3 from top to bottom, were separated with substrate immersing zymography. Different substrate preferences were observed: activities EV-1 and EV-2 were detectable with gelatin and casein substrates, respectively, while EV-1 and EV-2 had apparently stronger activities to gelatin than EV-3. Furthermore, activity EV-3 was only observed when gelatin was used as the substrate. The inhibitor sensitivities of EV-2 and EV-3 were similar from zymogram that they were inhibited by serine protease inhibitor PMSF but not by chelators OP. Moreover, EV-1 was a wide band on gelatin immersion zymography and completely inhibited by OP which indicated that EV-1 was a typically metalloprotease (Fig. 4A). These results showed that gelatin allowed a more sensitive detection of bacterial extracellular proteases and was consistent with Marokházi's research conclusion [21].

Folin phenol caseinolytic analyses showed that the total activity of all components from Vibrio $s p$. SQS2-3 was inhibited about $37 \%$ by OP and $86 \%$ by PMSF, respectively. The image quantitative analysis of all the bands, the total caseinolytic activity of Vibrio $s p$. SQS2-3 had been inhibited about $50 \%$ by OP and $77 \%$ by PMSF, respectively. The image quantitative analysis of gelatinolytic activity of Vibrio $s p$. SQS2-3 revealed significant different. There were about 40\% residual activity and $63 \%$ residual activities present $1 \mathrm{mM} \mathrm{OP}$ and $1 \mathrm{mM}$ PMSF respectively.
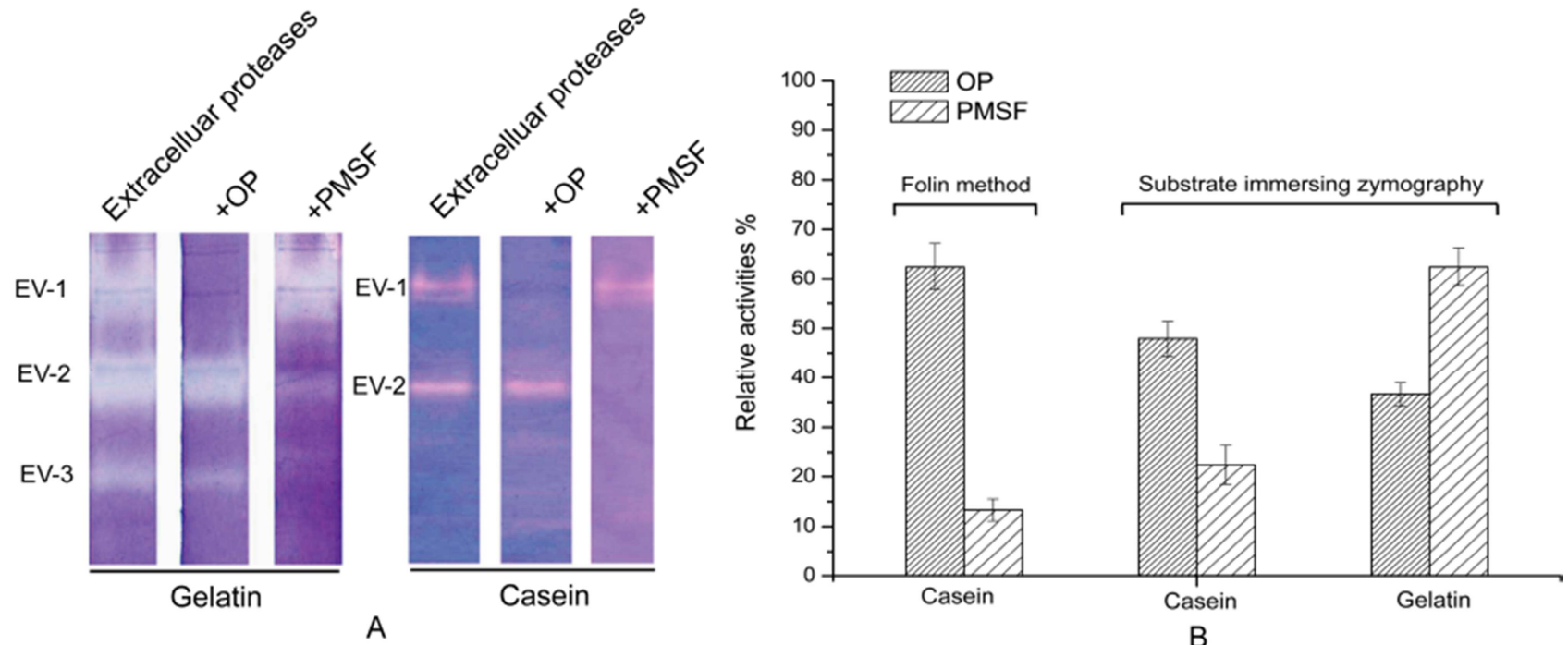

Fig. 4. The effect of inhibitors on proteases from the crude extract of Vibrio sp. SQS2-3. (A) Inhibitor-substrate immersing zymographic detection of Vibrio sp. SQS2-3 proteases in culture. (B) Compared analysis inhibition of proteases from the crude extract of Vibrio sp. SQS2-3 by OP or PMSF using Folin phenol method and inhibitor-substrate immersion zymography method. 
From the above results it can be concluded that the changes in the residual activities of crude enzyme (reflected by bands gray values) are expected to be indicators of the inhibitors dependence of the corresponding enzyme component. Thus, the residual activities were easily determined by the quantitative extraction of the gray values of bands. In traditional method, the crude proteases from bacteria were pre-incubated with $1 \mathrm{mM}$ PMSF or $1 \mathrm{mM}$ OP, respectively. After incubation, the protease activity of every sample was measured caseinolytic activity by Folin phenol method [15]. In this study, we found that some proteases cannot be detected on casein immersion zymography but can be detected on gelatin immersion zymography. The different sensitivities to the substrate and inhibitor indicate that different components have different substrate specificity. Thus, the inhibition rate of OP or PMSF should not be simply calculated according to the Folin phenol method, which is not as reliable as zymography to determine the activity of some enzymes. In our study, the enzymatic activities of each active component to different substrates were demonstrated by performing the inhibitor-substrate immersion zymography. The gel strips were soaked simultaneously in the substrate solution with different inhibitor. The changes in activity of the enzyme caused by inhibitors were easily determined by the quantitative extraction of the gray values of each band. So utilizing inhibitor-substrate immersion zymography, we can relatively accurate determine the specific activity or inhibition rate of every component of extracellular proteases with different substrates. In short, inhibitor-substrate immersion zymography is more suitable for in situ, rapid, sensitive and accurate detection of the diversity, variety and activity of bacterial extracellular proteases.

\subsection{Mass Spectrum Identification of Protease EV-2 and EV-3 from Vibrio sp. SQS2-3}

We also detected the characterization of purified protease EV-2 and EV-3 from Vibrio sp. SQS2-3 using mass spectrometry. These proteins were excised from the polyacrylamide gel and digested in situ with trypsin, and the resulting peptide mixture was eluted from the gel. An aliquot of this tryptic peptide mixture was analyzed by online microcapillary HPLC coupled with electrospray ionization-mass spectrometry. Sequences of isolated tryptic peptides were determined by semi-automated tandem mass spectrometry coupled with protein database searching. The results were shown in table 2. Protease EV-2 and EV-3 showed high sequence identity with alkaline serine protease asp which was originally identified as a virulence factor of Vibrio alginolyticus isolated from moribund Epinephelus coioides in an outbreak of vibriosis in a mariculture farm [22]. These results suggested that protease EV-2 and EV-3 originated from the same protein, based on the protein sequence found in the NCBI database, accession number ACC94160.1. And the serine protease result was consistent with the conclusion obtained from inhibitor-substrate immersion zymography (Fig. 4A).

In addition, we also found that the EV-2 and EV-3 should be the same kind of protease but with different molecular weight. Maybe protease EV-3 was generated through autoproteolytic removal of the terminal peptide. Autoproteolytic is a regulatory mechanism for controlling the activity of proteases. Autoproteolysis of protease was observed during sample preparation which the large peptides could autolyze further into smaller peptides 23]. The mature mechanism of this protease need to be further studied.

Table 2. Amino acid sequence of the protease by using NanoLC-ESI-MS/MS system.

\begin{tabular}{|c|c|c|c|c|c|}
\hline Protease & Peptide Mass & Peptide Sequence & Sequence Header & Similarity (\%) & Mr (calc) \\
\hline \multirow{4}{*}{ EV-3 } & 1166.6 & ${ }^{534}$ TGHGLVNAEAAK $^{546}$ & alkaline serine protease [Vibrio alginolyticus] & 100 & \multirow{4}{*}{$35 \mathrm{kDa}$} \\
\hline & 1542.84 & ${ }^{516}$ ALVKTATDIDVAGR $^{531}$ & alkaline serine protease [Vibrio alginolyticus] & 100 & \\
\hline & 1563.79 & ${ }^{335}$ LSDITLNGVSQFDR $^{349}$ & alkaline serine protease [Vibrio alginolyticus] & 100 & \\
\hline & 2865.43 & ${ }^{425}$ AAIVYSNSELPGLQNPFLVDTNNAYR ${ }^{451}$ & alkaline serine protease [Vibrio alginolyticus] & 100 & \\
\hline \multirow{8}{*}{ EV-2 } & 1166.6 & ${ }^{534}$ TGHGLVNAEAAK $^{546}$ & alkaline serine protease [Vibrio alginolyticus] & 100 & \multirow{8}{*}{$45 \mathrm{kDa}$} \\
\hline & 1189.6 & ${ }^{579}$ SSGAISAVEVDR ${ }^{590}$ & alkaline serine protease [Vibrio alginolyticus] & 100 & \\
\hline & 1563.79 & ${ }^{335}$ LSDITLNGVSQFDR $^{349}$ & alkaline serine protease [Vibrio alginolyticus] & 100 & \\
\hline & 1628.78 & ${ }^{219}$ VFNESGWGYSSGLVK ${ }^{233}$ & alkaline serine protease [Vibrio alginolyticus] & 100 & \\
\hline & 2190.99 & ${ }^{638}$ NTFQADFSGFESQGTWELK ${ }^{656}$ & alkaline serine protease [Vibrio alginolyticus] & 100 & \\
\hline & 2306.1 & ${ }^{591}$ SGDSGTVSIDVDISHTYIGDLR ${ }^{612}$ & alkaline serine protease [Vibrio alginolyticus] & 100 & \\
\hline & 2480.25 & ${ }^{613}$ VTLTSPTGGEVVLHNNTGGSANDIK ${ }^{637}$ & alkaline serine protease [Vibrio alginolyticus] & 100 & \\
\hline & 2865.43 & ${ }^{425}$ AAIVYSNSELPGLQNPFLVDTNNAYR ${ }^{451}$ & alkaline serine protease [Vibrio alginolyticus] & 100 & \\
\hline
\end{tabular}

\section{Conclusion}

The diversity of the bacterial extracellular proteases was usually investigated with protease inhibitors. PMSF (serine protease inhibitor), OP (metalloprotease inhibitor) were used to inhibit the activities of the proteases secreted by the screened strains for identification of these proteases. For the investigation of secreted proteolytic activities, we screened the cultures of the heterotrophic bacteria (i.e., Pseudolateromonas, Vibrio, and Bacillus) using inhibitor-substrate immersion zymography which was able 
to resolve activities according to their molecular properties. The inhibitor-substrate immersion zymography not only can be used to detect activity of various kinds of enzyme in multienzyme systems, but also can be used as a classification tool of enzyme. Our results indicated that the Folin phenol method, commonly used to measuring protease activity, was not comprehensive and accurately reflecting the protease activity. Maybe it was more conveniently and accurately to detect the enzyme activity and characterize its properties in situ by zymography band's gray value. In fact, some strains can produce an extracellular proteolytic complex formed by several proteases with proteolytic activity, which are important for the growth of bacteria through supplying cells with amino acids and nutrients [24]. Marine microorganisms show high diversity and can be a good source for isolating novel proteolytic enzyme [25]. Therefore the sensitive and quick detection of extracellular enzyme by inhibitor-substrate immersion zymography will be helpful to analyze the nature and characteristics of marine bacterial proteases.

\section{Acknowledgements}

The work was supported by National Natural Science Foundation of China (31070061, 31370104), Hunan Provincial Natural Science Foundation of China (13JJ9001), National Sparking Plan Project (2013GA770009) Shandong Provincial Natural Science Foundation (ZR2014CQ043), Open-End Fund for the Valuable and Precision Instruments of Central South University, and Fundamental Research Funds for the Central Universities of Central South University.

\section{References}

[1] Barrett, A. J., N. D. Rawlings, and J. F. Woessner (2003) The handbook of proteolytic enzymes, $2^{\text {nd }}$ ed. Academic Press.

[2] Rao, M. B., M. S. Tanksale, M. S. Ghatge, and V. V. Deshpande (1998) Molecular and biotechnological aspects of microbial proteases. Microbiol. Mol Biol. Rev. 62: 597-635.

[3] Rawlings, N. D., A. J. Barrett, and A. Bateman (2014) Using the MEROPS database for proteolytic enzymes and their inhibitors and substrates. Curr. Protoc. Bioinformatics. 48: 1.25.1-1.25.33.

[4] Foukis, A., P. Y. Stergiou, L. G. Theodorou, M. Papagianni, and E. M. Papamichael (2012) Purification, kinetic characterization and properties of a novel thermo-tolerant extracellular protease from Kluyveromyces marxianus IFO 0288 with potential biotechnological interest. Bioresour. Technol. 123: 214-220.

[5] Vary, P. S., R. Biedendieck, T. Fuerch, F. Meinhardt, M. Rohde, W. D. Deckwer, and D. Jahn (2007) Bacillus megaterium-from simple soil bacterium to industrial protein production host. Appl. Microbiol. Biotechnol. 76: 957-967.

[6] Hunter, E. M., H. J. Mills, and J. E. Kostka (2006) Microbial community diversity associated with carbon and nitrogen cycling in permeable shelf sediments. Appl. Environ. Microbiol. 72: $5689-5701$.
[7] Ran, L. Y., H. N. Su, M. Y. Zhou, L. Wang, X. L. Chen, B. B. Xie, X. Y. Song, M. Shi, Q. L. Qin, X. Pang, B. C. Zhou, Y. Z. Zhang, and X. Y. Zhang (2014) Characterization of a novel subtilisin-like protease myroicolsin from deep sea bacterium Myroides profundi D25 and molecular insight into its collagenolytic mechanism. J. Biol. Chem. 289: 6041-6053.

[8] Van Dijl, J. M. and M. Hecker (2013) Bacillus subtilis: from soil bacterium to super-secreting cell factory. Microb. Cell. Fact. 12: 3 .

[9] Zacaria, J., A. P. L. Delamare, S. O. P. Costa, and S. Echeverrigaray (2010) Diversity of extracellular proteases among Aeromonas determined by zymogram analysis. J. Appl. Microbiol. 109: 212-219.

[10] Massaoud, M. K., J. Marokházi, A. Fodor, and I. Venekei (2010) Proteolytic enzyme production by strains of the insect pathogen xenorhabdus and characterization of an early-log-phase-secreted protease as a potential virulence factor. Appl. Environ. Microbiol. 76: 6901-6909.

[11] Li, X. and H. Y. Yu (2012) Purification and characterization of novel organic-solvent-tolerant $\beta$-amylase and serine protease from a newly isolated Salimicrobium halophilum strain LY20. FEMS. Microbiol. Lett. 29: 204-211.

[12] Liew, S. M., S. T. Tay, and S. D. Puthucheary (2013) Enzymatic and molecular characterisation of leucine aminopeptidase of Burkholderia pseudomallei. BMC. Microbiol. 13: 110.

[13] Liu, D., X. H. Yang, J. F. Huang, R. B. Wu, C. L. Wu, H. L. $\mathrm{He}$, and $\mathrm{H}$. Li (2015) In situ demonstration and characteristic analysis of the protease components from marine bacteria using substrate immersing zymography. Appl. Biochem. Biotechnol. 175: 489-501.

[14] He, H. L., J. Guo, X. L. Chen, B. B. Xie, X. Y. Zhang, Y. Yu, B. Chen, B. C. Zhou, and Y. Z. Zhang (2012) Structural and functional characterization of mature forms of metalloprotease E495 from arctic Sea-Ice bacterium pseudoalteromonas sp. SM495. PLOS. ONE. 7: e35442.

[15] Zhou, M. Y., X. L. Chen, H. L. Zhao, H. Y. Dang, X. W. Luan, X. Y. Zhang, H. L. He, B. C. Zhou, and Y. Z. Zhang (2009) Diversity of both the cultivable protease-producing bacteria and their extracellular proteases in the sediments of the South China sea. Microb. Ecol. 58: 582-590.

[16] Shevchenko, A., M. Wilm, O. Vorm, and M. Mann (1996) Mass spectrometric sequencing of proteins silver-stained polyacrylamide gels. Anal. Chem. 68: 850-858.

[17] Zhang, X. M., N. Liu, F. Yang, J. H. Li, L. S. Wang, G. J. Chen, and P. J. Gao (2012) In situ demonstration and quantitative analysis of the intrinsic properties of glycoside hydrolases. Electrophoresis. 33: 280-287.

[18] Xie, B. B., Y. L. Shu, Q. L. Qin, J. C. Rong, X. Y. Zhang, X. L. Chen, M. Shi, H. L. He, B. C. Zhou, and Y. Z. Zhang (2012) Genome sequences of type strains of seven species of the marine bacterium Pseudoalteromonas. J. Bacteriol. 194: 2746-2747.

[19] Haddar, A., R. Agrebi, A. Bougatef, N. Hmidet, A. Sellami-Kamoun, and M. Nasri (2009) Two detergent stable alkaline serine-proteases from Bacillus mojavensis A21: purification, characterization and potential application as a laundry detergent additive. Bioresour. Technol. 100: 3366-3373. 
[20] Sinha, R., A. K. Srivastava, and S. K. Khare (2014) Efficient proteolysis and application of an alkaline protease from halophilic Bacillus sp. EMB9. Prep. Biochem. Biotechnol. 44: 680-696.

[21] Marokházi, J., K. Lengyel, S. Pekár, G. Felföldi, A. Patthy, L. Gráf, A. Fodor, and I. Venekei (2004) Comparison of proteolytic activities produced by entomopathogenic Photorhabdus bacteria: strain- and phase-dependent heterogeneity in composition and activity of four enzymes. Appl. Environ. Microbiol. 70: 7311-7320.

[22] Rui, H., Q. Liu, Q. Wang, Y. Ma, H. Liu, C. Shi, and Y. Zhang (2009) Role of alkaline serine protease, asp, in vibrio alginolyticus virulence and regulation of its expression by
luxO-luxR regulatory system. J. Microbiol. Biotechnol. 19: 431-438.

[23] Miyoshi, I. M., Wakae, H., Tomochika, K. I., and Shinoda, S. 1997. Functional domains of a zinc metalloprotease from Vibrio vulnificus. J. Bacteriol. 179 (23): 7606-7609.

[24] Sinsabaugh, R. L. and J. J. Shah (2010) Integrating resource utilization and temperature in metabolic scaling of riverine bacterial production. Ecology. 91: 1455-1465.

[25] Wang, Y., A. Nakajima, K. Hosokawa, A. B. Soliev, I. Osaka, R. Arakawa, and K. Enomoto (2012) Cytotoxic prodigiosin family pigments from Pseudoalteromonas sp. 1020R isolated from the Pacific coast of Japan. Biosci. Biotechnol. Biochem. 76: 1229-1232. 